\title{
IDENTIFICATION OF THE REFRIGERANT PRESSURE IN SPLIT-TYPE AIR CONDITIONERS BASED ON HARMONIC ANALYSES OF ELECTRICITY SUPPLY CURRENT
}

\author{
Zeljko Djurisic, Milenko Djuric \\ University of Belgrade, Faculty of Electrical Engineering \\ Bulevar kralja Aleksandra 73, 11000 Belgrade \\ Serbia \\ djruisic@etf.bg.ac.yu; mdjuric@etf.bg.ac.yu;
}

\begin{abstract}
An analysis is presented of the interdependence between power supply characteristics of single phase split-type air conditioners and pressure of the operating fluid (refrigerant) within the air conditioner system. On the basis of the tests performed by a real air conditioner the corresponding correlations have been established. Of special interest is the influence of refrigerant pressure on the level of the second harmonic of the supply current and active input power of air conditioner. The established correlations allow that by processing the input current and voltage of an air conditioner one can make a diagnosis of the state of refrigerant pressure within the system which is a fast, comfortable, and ecologically better approach compared to the classic direct measurement on the operating fluid by a pressure gauge.
\end{abstract}

\section{KEY WORDS}

Identification, ir conditioners; refrigerant pressure; power supply; harmonic analyses.

\section{Introduction}

An ever increasing number of air conditioners, particularly in urban residential and office objects, in Serbia is being installed over the past decade, Fig. 1. Typically, the units of rated power $12000 \mathrm{BTU} / \mathrm{h}$ of the split-type system are being in use. HFC refrigerants such as R410A, R407C and HCFC22 (in older types) predominantly use in residential and office airconditioners.

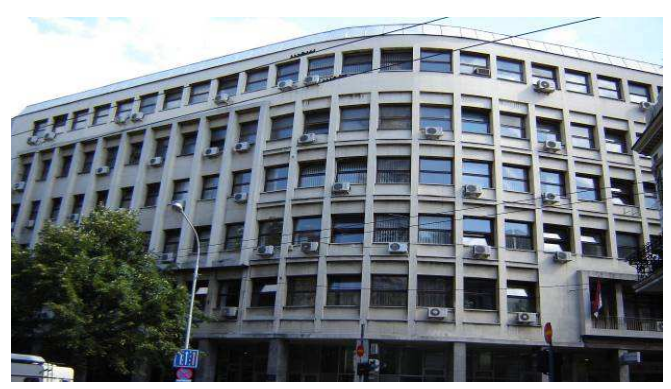

Fig. 1: An office building in Belgrade having many single phase split-type air conditioners installed
The outdoor units of air conditioners are often mounted on building walls at places which are difficult to access [1], and their maintenance is sometimes a difficult and hazardous job. The most frequent problem is to make a diagnosis of the refrigerant pressure in the system. Measurement of refrigerant pressure is usually performed by a classic pressure gauge connected to the corresponding valve on the outdoor unit of air conditioner. Owing to the difficulties in accessing these outdoor units the maintenance job could cause serious and hazardous problems to maintenance personnel. In addition, during each measurement certain amount of refrigerant [2] leaks in the atmosphere contributing to the negative global effects on the atmosphere $[3,4]$.

In this article a new possibility of detecting the state of refrigerant pressure on the basis of an analysis of electrical parameters of the corresponding power supply is presented. In this way the diagnosis of the state of refrigerant pressure would be made by performing measurements at the electrical inlet of air conditioner which is a fast, comfortable, less hazardous, and ecologically superior approach compared to the classical measurement of refrigerant pressure by a pressure gauge. In addition to the mention good points, the proposed method belongs to the group of non-invasive measurement methods since the measurement does not involve contact with the refrigerant whose pressure is being measured.

\section{The Measurement Method}

For the purpose of establishing how the state of refrigerant pressure affects the current and power of the supply, a test has been performed with a typical air conditioner of rated power $12000 \mathrm{BTU} / \mathrm{h}$. The principle wiring diagram of the measurement is shown in Fig. 2.

The measurement of electrical characteristics of air conditioner power supply and harmonic analyses of current has been carried out by a PC based measurement system [5]. 
Refrigerant pressure measurement was performed by a pressure gauge at the corresponding connection on the outer component. The use is made of a vacuum pump for refrigerant evacuation from the system to achieve pressures below atmospheric pressure.

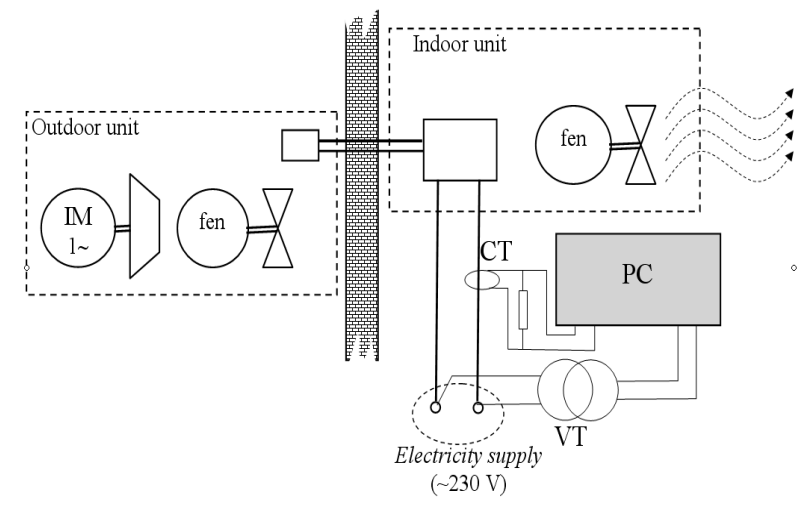

Fig. 2: The principle wiring diagram of the analyzer of the current and voltage waveforms of air conditioners

Notation in Fig. 2 is:

VT - voltage measuring transformer,

CT - inductively coupled current measuring transformer having a built-in resistor, and $\mathrm{m}$ - pressure gauge.

\section{The Measurement Results}

During the measurements the air conditioner was operating in the cooling mode. The refrigerant was allowed to leak causing a drop of refrigerant pressure which was measured by a pressure gauge. The pressure in the system prior to leaking was 3 bar. The leaking of the refrigerant was organized so that the pressure was changing from 3 bar to 0 in steps of 0.5 bar. At each pressure the air conditioner was operating for $2 \mathrm{~min}$ in order to allow that the pressure within the system becomes stable. During this process of refrigerant leakage the supply current and its harmonic distortions were measured together with the active and non-active powers of the power supply.

Fig. 3 shows the variation of the r.m.s. value of the supply current during the experiment. The variation of the supply current was caused by variation (reduction) of the power of the compressor. Fig. 4 shows the variations of the active and non-active powers of the power supply during the experiment. The dependence of the active power of the air conditioner on the pressure within the system is clearly visible. In the course of variation of the refrigerant pressure from 3 bar to $0 \mathrm{bar}$, the power of the examined air conditioner dropped from $1020 \mathrm{~W}$ to $680 \mathrm{~W}$. The nonactive power also dropped to some extent since owing to the reduction of the current the stray flux was reduced, whereas the magnetizing flux, which is related to the voltage, remained unchanged.

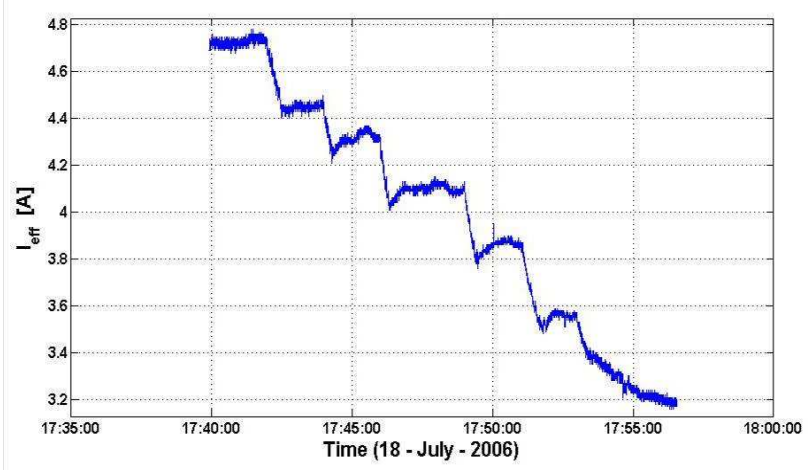

Fig. 3: Variation of the r.m.s. value of the supply current during refrigerant leakage from 3 bar to 0 bar in steps of 0.5 bar

The drop of non-active power at the end of the experiment, which can be noted in the diagram of Fig. 4, was caused by a drop of the voltage in the supply network. Fig. 5 shows the variation of the supply voltage in the course of the experiment.

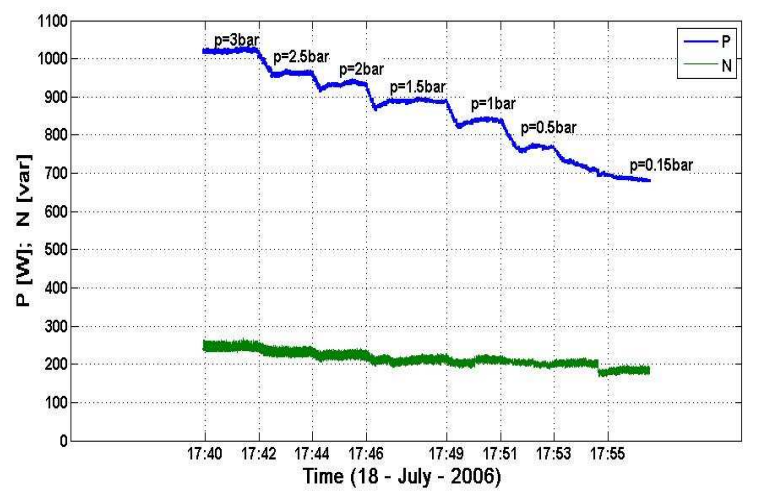

Fig. 4: The variations of the active and non-active powers in the course of reduction of the refrigerant pressure in the system

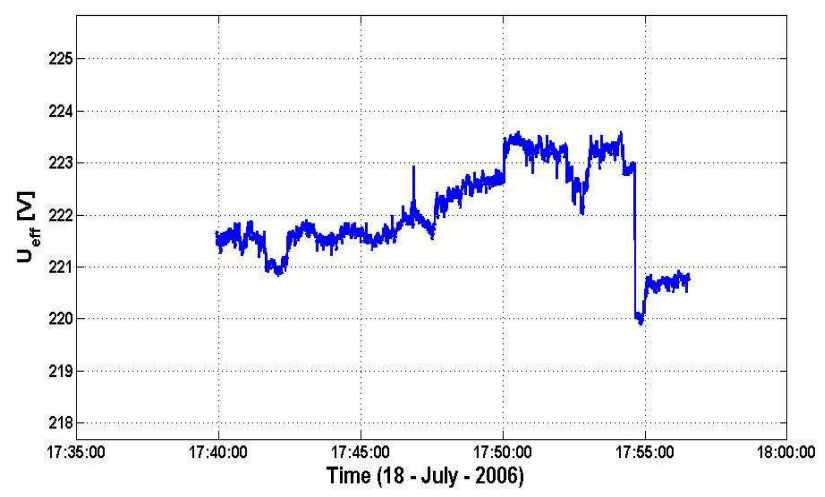

Fig. 5: The variation of the r.m.s. value of the supply voltage during the experiment 
Fig. 6 shows the variation in time of the percentage level of higher harmonics in the supply current of the air conditioner.

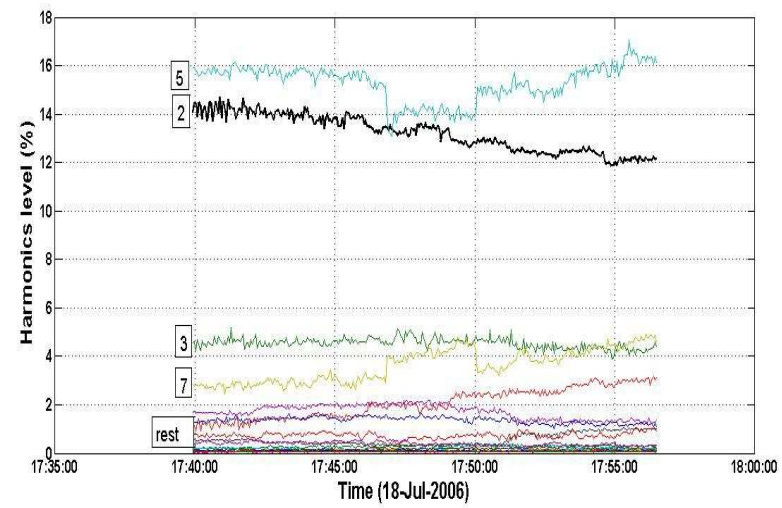

Fig. 6: Time variation of the higher harmonics of the supply current during the experiment

The second harmonic of the supply current, which is caused by the operation of the compressor, is of particular interest for this analysis. Reduction of the refrigerant pressure lowers the percentage level of the second harmonic. In this case this reduction amounts to approximately $2 \%$, but it becomes noticeable only when the refrigerant pressure falls below 2 bar. The reason is that the total r.m.s. value of the current of the fundamental harmonic reduces and the variation of the percentage of the second harmonic is small. At the pressure of 0 bar there is still certain power and also the second harmonic of the current. This is a consequence of the fact that even at this state there is still some refrigerant in the system. In order to show that the second harmonic is exclusively the consequence of the existence of the refrigerant in the system, the refrigerant was evacuated by means of a vacuum pump. Fig. 7 shows the spectrum of harmonics in the supply current of an air conditioner where the refrigerant was completely evacuated.

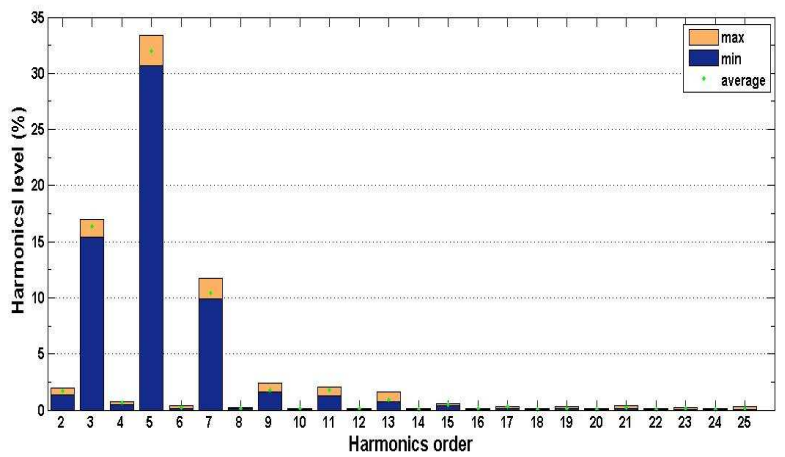

Fig. 7: The spectrum of higher harmonics of the supply current of an air conditioner where the refrigerant was completely evacuated
This test and Fig. 6 demonstrate that the second harmonic is exclusively the consequence of operation of the compressor and that there is a direct relation between the level of second harmonic and level of refrigerant pressure in the system. The second harmonic of the supply current is practically non-existent if there is no refrigerant in the system. Certain small value (approximately $2 \%$ ) in Fig. 7 is the consequence of the fact that absolute vacuum in the system can not be achieved and, also, it could be a consequence of the presence of the second harmonic of the supply voltage.

Fig. 8 shows the dependence of the levels of absolute value of the second harmonic of the supply current and active power of air conditioner on the refrigerant pressure within the system.

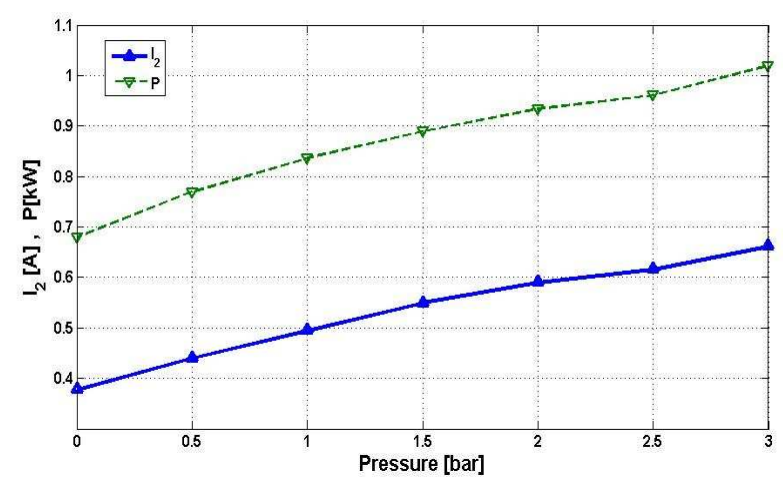

Fig. 8: The measured levels of active power $(P)$ and r.m.s value of the second harmonic $\left(I_{2}\right)$ of the supply current of an air conditioner as functions of the refrigerant pressure in the system

This analysis shows that it is possible to make a diagnosis of the refrigerant pressure on the basis of the measurement of active power and level of the second harmonic. In the specifications of each air conditioner model the rated active power is listed. Therefore, by measuring the active power of the supply one can make a rough assessment of the refrigerant pressure in air conditioner. If this analysis is to be of some quantitative value, it is necessary to take into account the dependence of the refrigerant pressure on temperature. Therefore, ambient temperature should be taken as an independent variable. Since any deviation of the active power from its rated value can be caused by a fault in some of the system components or by an increase of the friction in the ball bearings of the motor, it is recommended that a diagnosis of the state of refrigerant pressure is performed by measuring both active power and second harmonic of the supply current. By a comparative measurement of the power and level of the second harmonic of the supply current, one can make a quite reliable conclusion as regards the state of refrigerant pressure in an air conditioner. For each air conditioner model the reference diagrams, like the ones shown in Fig. 8, could be made in the corresponding service organization. By comparing 
field data with the corresponding reference diagrams one can make an assessment of the state of refrigerant pressure in the system.

\section{Conclusion}

The pressure of the operating fluid in an air conditioner directly affects the level of active power and spectrum of harmonics of the supply current. The manufacturing or service organization can pe4rform laboratory measurements of the reference values of the active power and level of the second harmonic of the supply current as functions of the refrigerant pressure in the system for each air conditioner model. Comparing field measurements with the corresponding reference values allows making quite a reliable assessment of the refrigerant pressure in the system. This approach offers a comfortable, fast, and reliable measurement of the refrigerant pressure. In addition, this indirect approach to the measurement is ecologically acceptable because there is no risk of any leakage of the very harmful refrigerant during performing tests of its pressure. The measuring equipment used for performing this work consists of a lap top computer and voltage and current measuring transformers, i.e. it is inexpensive and readily available.

\section{References}

[1] A. Avara, E. Daneshgar, Optimum placement of condensing units of split-type air-conditioners by numerical simulation, Energy and Buildings 40, 2008, 1268-1272.

[2] E. Preisegger, Automative air Conditioning impact of refrigerant on global warming, Proceedings of the Joint IPCC-TEAP Expert Meeting on Options for the Limitation of Emissions of HFCs and PFCs, Petten, Netherlands, 1999.

[3] W. Chen, A comparative study on the performance and environmental characteristics of R410A and R22 residential air conditioners, Applied Thermal Engineering 28, 2008, 1-7.

[4] Y. Xuan and G. Chen, Experimental study on HFC161 mixture as an alternative refrigerant to R502, International Journal of Refrigeration 28, 2005, pp. 436441.

[5] Ž. Djurišić, M. Djurić, Description of the measurement-acquisition system for quality control of electric power in a distribution system, Proceedings of the $27^{\text {th }}$ Symposium CIGRE, Zlatibor 2005. (in Serbian) 